\title{
Production of the Sesquiterpenoid, Juvenile Hormone-1 (JH-I), and of Vitamin E in the Accessory Sexual (colleterial) Glands of Adult Male Moths, Hyalophora cecropia (Linnaeus, 1758), (Lepidoptera: Saturniidae) ${ }^{1}$
}

\author{
Michal Paroulek ${ }^{2}$ and Karel Sláma ${ }^{3}$
}

\begin{abstract}
The content of the sesquiterpenoids, juvenile hormones (JH), JH-I, JH-II, JH-III and Vitamin $\mathrm{E}(\alpha-, \beta-, \gamma$ - and $\delta$-Tocopherols) was investigated in mated and unmated adult Cecropia silkworms, Hyalophora cecropia (Linnaeus, 1758), using combined chromatography/mass spectrometry methods. The relatively most abundant derivative of Vitamin E was $\alpha$-tocopherol, the highest concentrations of which were found in the fat body of unmated males (30 ng/organ), and which was substantially decreased after mating. Conversely, the content of $\alpha$-tocopherol increased more than twice in female abdomens after mating. This suggests that Vitamin E was transferred from the male to the female reproductive systems during courtship. Chromatographic analysis of extracts prepared from female abdomens, male fat bodies, and male reproductive organs revealed that separate fractions of Vitamin E ( $\alpha$-tocopherol, $\beta$ - $+\gamma$-tocopherols and $\delta$-tocopherol) were evenly distributed in quantitative proportions of $18400: 550: 100$, respectively. These results suggest that sesquiterpenoid derivatives of Vitamin E play important functions in insect reproduction, in analogy with similar functions of Vitamin E during reproduction of vertebrate animals.

The sesquiterpenoid 10,11-epoxy-homofarnesoate ester known as JH-I, which is thought to be the true hormone of insect corpus allatum, was virtually absent or undetectable in abdomens of virgin females of Cecropia moths. In contrast to Vitamin E, JH-I was also absent or occurred below the detectable limits in fat body/haemolymph fractions of adult males. Actually, the sesquiterpenoid JH-I was found exclusively in the lumen of the exocrine, accessory sexual (colleterial) glands of unmated males (50 ng/organ). Smaller amounts of this JH-I excretory product occurred also in the associated parts of the male reproductive system (testes, vas deferens and ejaculatory ducts), which are directly connected with the colleterial gland by the connecting ducts. The content of JH-I in the colleterial glands and ejaculatory ducts decreased to 9 ng/organ after courtship, when JH-I conversely appeared in the female abdomens $(20 \mathrm{ng} / \mathrm{abdomen})$. We conclude, therefore, that both these sesquiterpenoid compounds, JH-I and Vitamin E, become translocated from males into the female reproductive system by ejaculate during the courtship. A possibility that both Vitamin E and JH-I serve important physiological functions (antioxidant, sperm survival, fertilisation, stabilisation of egg chorion) has been suggested. The relative contents of JH-I to JH-II to JH-III in the colleterial glands revealed proportions of 64000 to 100 to 20, respectively. According to these data, the most abundant and widespread $\mathrm{JH}$ analogue of other insect species, methyl epoxyfarnesoate, commonly known as JH-III, was present only in traces. The real physiological status of JH-I in adult Cecropia has been defined as the excretory product of the exocrine colleterial gland. The presence of JH-I only in the exocrine colleterial glands of males and not in the females, combined with the secretory inactivity of the corpora allata, led us to formulate several physiological reasons why JH-I, as an excretory product of exocrine glands cannot be considered an insect hormone. A possibility that the true hormone of insect corpus allatum is still unknown has been emphasized.
\end{abstract}

\footnotetext{
${ }^{1}$ Submitted on April 30, 2014 . Accepted on June 6, 2014. Last revisions received on July 5, 2014.

${ }^{2}$ Biology Centre of Czech Academy of Sciences, Institute of Entomology, Branišovská 31, 50700 Č. Budějovice, Czech Republic. E-mail: paroulek@gmail.com

${ }^{3}$ Biology Centre of Czech Academy of Sciences, Institute of Entomology, Drnovská 507, 16100

Praha 6, Czech Republic. E-mail: $\underline{\text { slama@entu.cas.cz }}$
}

DOI: 10.9784/LEB2(2)Paroulek.01

Electronically available on July 18, 2014. Mailed on July 16, 2014. 
Key Words: Juvenile hormone $(\mathrm{JH})$, corpus allatum hormone (CAH), JH-active extracts, JH analogues (juvenoids), accessory sexual glands, JH-I excretory product, JH-I in male ejaculate, tocopherols (Vitamin E), sesquiterpenoids in reproduction, Lepidoptera: Saturniidae, Hyalophora cecropia

\section{Introduction}

A recent review over a 50-year history of insect juvenile hormone analogues reveals two alternative theories to explain hormonal control of insect development (Sláma, 2013). The first is represented by a generally approved and widely used theory of Gilbert-Riddiford (Schneiderman and Gilbert, 1964; Nijhout, 1994; Nation, 2002; Klowden, 2007; Gilbert, 2009; Riddiford, 1996; Devillers, 2013a), which proposes that insect development is regulated by a moulting hormone secreted from the prothoracic gland $(\mathrm{PG})$ in response to a prothoracicotropic hormone (PTTH) from the brain (Riddiford, 2012; Jindra et al., 2013). The theory also proposes that larval, pupal and adult moult cycles depend on the respectively large, medium or zero concentrations of a sesquiterpenoid JH-I secreted from the endocrine gland, corpus allatum. The outline of Gilbert-Riddiford theory can be seen in Figure 1. The second, less renowned and antagonistic theory of Novák-Sláma (Novák, 1959, 1966, 1967; Sláma, 1962, 1971, 1985, 1999) proposes that PG is used for regulation of physiological functions other than stimulation of moults. These glands are subordinated targets of corpus allatum hormone, not PTTH. They are used for production of metabolic water by complete combustion of dietary lipids during the larval feeding periods (Sláma and Lukáš, 2013). Regulation of larval growth and metamorphosis is determined by the minimum physiologically effective concentrations of the corpus allatum hormone. The progress in successive moulting cycles is achieved by peptidic or proteinaceous hormones released from the central neuroendocrine system (neurosecretory cells of the brain, corpora cardiaca and corpora allata), not by a mysterious moulting hormone from PG (reviewed by Sláma, 2013).

The first materials with juvenile hormone $(\mathrm{JH})$ activity were discovered by Williams in 1947 (see Williams, 1952). The activity was found in lipid extracts made from abdomens of adult male Hyalophora cecropia silkworms. Obviously, Williams did not know at the very beginning of $\mathrm{JH}$ research about the future discovery of as many as 4000 structurally unrelated chemical mimics of $\mathrm{JH}$ (juvenoids; Sláma et al., 1974; Sláma, 1999). He reasonably assumed that the JH-active material in the lipid extracts should be made within the corpus allatum. Later, when large depots of $\mathrm{JH}$ were found in the male accessory sexual glands, he expected synthesis of the active compound within the corpus allatum and its subsequent transport and accumulation in the male accessory sexual glands (Williams, 1956, 1959, 1960, 1963). 


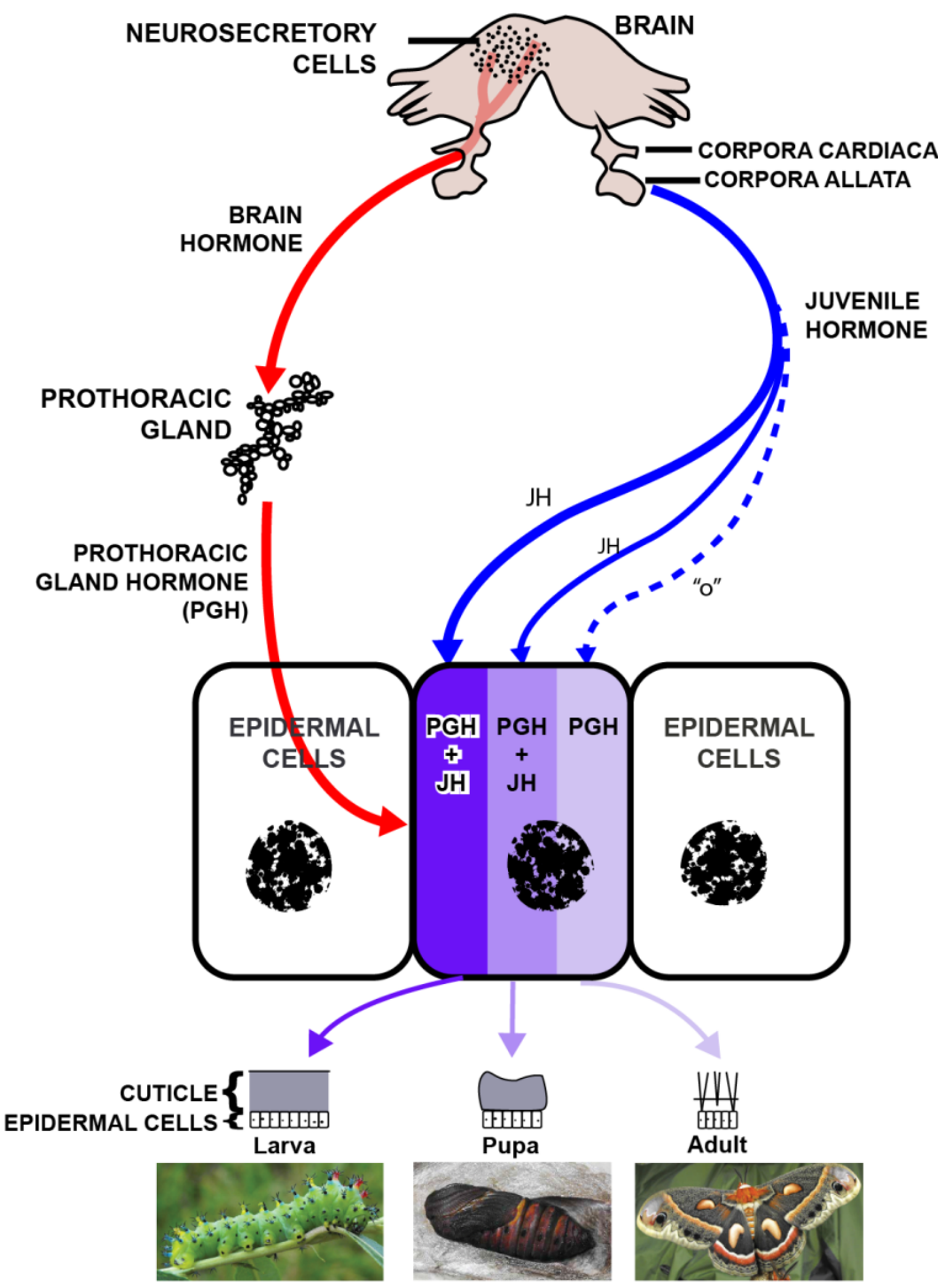

Figure 1. Schematic diagram of hormonal theory of insect metamorphosis, proposed more than 50 years ago by Schneiderman and Gilbert $(1958,1964)$ after modification of Wigglesworth (1957) model based on the juvenile hormone theory of Piepho. The larval, pupal or adult developmental programme was determined in each epidermal cell by the respectively large, medium or zero concentrations of juvenile hormone. The moult cycles were stimulated by the moulting hormone released from the prothoracic glands in response to brain hormone. Reproduced from Sláma (2013), with permission from Brill Publishers, Leiden, The Netherlands. 
The first JH-active molecule with a determined chemical structure was the sesquiterpenoid alcohol farnesol, isolated from the lipid extracts of yeast and excrements of the beetle Tenebrio molitor Linnaeus, 1758 (Coleoptera: Tenebrionidae) (Karlson and Schmialek, 1959; Schmialek, 1961). This finding immediately attracted the attention of biologists and chemists to the field of isoprenoids. Schmialek (1963) also analysed the lipid extracts of Cecropia and identified the JH-active compound as 3,7,11-trimethyl-2,6,10-tridecatrien-1-ol, which co-chromatographed with farnesol. Later, Röller and Bjerke (1965) and Röller et al. (1967) purified the lipid extracts of Cecropia and identified the most active compound as methyl 10,11-epoxy, 7-ethyl-3,11-dimethyl-2,6-tridecadienoate (Röller et al., 1967, 1969), which became generally known as JH-I. The related $\mathrm{JH}$-active compound, di-homofarnesoate, also found in the lipid extracts of Cecropia, became known as JH-II (Meyer et al., 1965, 1968).

The presence of JH-I in relatively large, male accessory sexual (colleterial) glands (Meyer et al., 1968; Shirk et al., 1976) and its virtual absence in females or males of other species represented a great puzzle for biochemists investigating the biosynthesis of JH-I. In agreement with Williams (1963), everybody believed that JH-I should be at first synthetised in the corpus allatum, then released into haemolymph and absorbed by different tissues and organs. According to extensive biochemical studies, the hormone was released from the corpus allatum in the form of the biologically inactive homofarnesoic acid, which was esterified and converted into the less polar and biologically active methyl ester, JH-I, in different tissues and organs (Peter et al., 1979, 1981; Shirk et al., 1983; Goodman and Granger, 2005). These conclusions were supported by extensive biochemical studies which confirmed in vitro esterification of farnesoic acid by $\mathrm{JH}$-specific O-methyltransferase enzymes (reviewed by Tobe and Stay, 1985; Hui et al., 2013).

A direct proof that JH-I is indeed the true corpus allatum hormone of $H$. cecropia does not exist. The conclusion depends mostly on a brief statement of Röller and Dahm (1970), who cultivated corpora allata in vitro and found JH-I in the medium. Later on, when relatively large depots of JH-I were found in the male accessory sexual glands (Meyer et al., 1968; Shirk et al., 1976; Peter et al., 1981), the identity problem was solved by tentative biosynthesis of the sesquiterpenoid $\mathrm{JH}$-precursor ( $\mathrm{JH}$-acid) in the corpus allatum, followed by its metabolism and accumulation in the accessory sexual glands. This biochemical explanation was, however, in serious conflict with the general rules of endocrinology, according to which animal hormone is never released from endocrine glands in inactive form and never accumulates or makes a depot in the peripheral target tissues (Hanc, 1959). The more recent suggestions about simultaneous biosynthesis of JH-I in both the corpus allatum and peripheral target tissues, such as occurs in male accessory sexual glands (De Loof et al., 2013, 2014), is also in conflict with general endocrinological knowledge. 
In 1961, endocrinologists investigating the effects of implanted corpus allatum (Novák, and Červenková, 1959; Sláma, 1961) compared the action of farnesol and the lipid extracts from Cecropia with the effects of the transplanted corpora allata. It appeared that the assays used for testing JH activity were largely nonspecific or could be completely false, because a number of trivial lipids, such as free fatty acids and fatty alcohols, exhibited the false "pseudojuvenilizing" or "JH-mimetic" effects (Sláma, 1961, 1962). Endocrinological arguments that the lipid extracts of Cecropia need not contain the true corpus allatum hormone (Sláma, 1962; Novák, 1966) were supported by evidence showing that the corpora allata of insects never secreted their hormone in a nonfeeding insect stage (reviewed by Pflugfelder, 1958; Novák, 1976). This fact is confirmed by the findings of Yamashita et al. (1961) and Banno and Akai (1988), who found that corpora allata of adult silkworms were virtually inactive.

After 1980, endocrinological aspects of JH research moved into the fields of biochemistry, molecular biology and industrial research directed to novel $\mathrm{JH}$ analogues used in insect control (reviewed by Sláma, 1985, 1999; Sláma et al., 1974). The total number of terpenoid or nonterpenic mimics of JH was estimated to more than 4000 compounds, some of which were million-fold more effective than JH-I (reviewed by Sláma, 1999). The relatively low hormonal activity of the sesquiterpenoid JH-I increased previous endocrinological doubts about identity of JH-I with the corpus allatum hormone (Sláma, 1961, 1962; Novák, 1966).

In this work we have reinvestigated the endocrine status of JH-I, hoping to resolve the physiological role of JH-I in the reproductive system of Hyalophora cecropia. In the previous study (Jedlička et al., 2009), we found production of acyl glycerols and Vitamin E in the female accessory sexual glands of the European fire bug, Pyrrhocoris apterus (Linnaeus, 1758) (Hemiptera: Pyrrhocoridae). These specifically female exocrine glands, whose growth and development is controlled by the hormone of the corpus allatum, produce Vitamin E with sesquiterpenoid side chains, exhibiting important antioxidant, antimicrobial, sperm protecting and fertilisation enhancing functions (Jedlička, 2011). After allatectomy, the females contained rudimentary ovaries and accessory sexual glands without sesquiterpenoid and other excretory products (Jedlička et al., 2009). The formation of sesquiterpenoid Vitamin E in female exocrine sexual glands of Pyrrhocoris apterus (Jedlička et al., 2009) provided strong presumptive evidence that the sesquiterpenoid $\mathrm{JH}-\mathrm{I}$, found in the male accessory sexual glands of taxonomically unrelated Hyalophora cecropia (Meyer et al., 1965; Shirk et al., 1976, 1981; Peter et al., 1981), could serve similar antioxidant or sperm protecting physiological functions. These calculations prompted us to reinvestigate the distribution and role of Vitamin E and JH-I in reproductive organs of adult Cecropia silkworms. The results are briefly described below. 


\section{Methods}

Adult males and females Hyalophora were obtained from diapausing pupae purchased from the dealers. After the obligatory period of pupal chilling at $5^{\circ} \mathrm{C}$, the adults were allowed to eclose at room temperature. Unfortunately, the amount of successfully eclosed moths was reduced to 4 males and 6 females, which were separated into two equal groups of mated and unmated specimens. We are aware of the limited number of experimental specimens. However, due to the relatively large qualitative differences between the results of individual experimental groups and due to general importance of the results, we describe the preliminary data here to avoid further delay. The male reproductive organs [testes, vas deferens, ejaculatory ducts, accessory sexual (colleterial) glands] were dissected 3 days after adult eclosion in insect Ringer solution, using conventional dissection methods. Due to fragility of the adult fat body, the samples of male fat body were removed together with the occluded haemolymph (estimated approximately to $25 \%$ on $\mathrm{v} / \mathrm{v}$ basis). The dissected organs of the males were homogenized in $3 \mathrm{ml}$ of chloroform : methanol mixture $(1: 2 \mathrm{v} / \mathrm{v})$. Female abdomens were isolated from previously anaesthetized specimens. Mated females were sacrificed before initiation of egg laying. Female abdomens were cut into smaller pieces and extracted in $6 \mathrm{ml}$ of the above indicated solvent mixture for 3 days, followed by centrifugation for $5 \mathrm{~min}$ at $10000 \mathrm{~g}$.

The extracts were evaporated on the Speed-Vac centrifuge, redissolved in 1 $\mathrm{ml}$ of 30\% methanol and then pre-purified on SPE columns (Oasis HLB, Waters Co., USA.). The columns were conditioned with $1 \mathrm{ml}$ of methanol, according to the recommendation of the producer, followed with $1 \mathrm{ml}$ of $30 \%$ methanol. After application of the sample, chromatographic columns were washed with 1 $\mathrm{ml}$ of $30 \%$ methanol and the analytes were eluted with $100 \%$ methanol. The eluate was dried on the Speed-Vac centrifuge and redissolved in the mobile phase used for analysis in the liquid chromatography-mass spectrometry method (20\% $\mathrm{MeOH}, 3 \%$ acetonitrile $(\mathrm{MeCN})$ in water buffered with $5 \mathrm{mM}$ ammonium formate).

Chemical analyses were made on LTQ quadrupole linear ion trap mass spectrometer (Thermo Electron Corp., USA) in APCI mode. Positive APCI spectra were recorded using $5 \mathrm{~mA}$ discharge current, $350{ }^{\circ} \mathrm{C}$ vaporizer temperature and $250^{\circ} \mathrm{C}$ capillary temperature. The extraction method, UPLC and MS/MS conditions were compatible with analysis of the tocopherols as well as JH-I to JH-III in a single run. Specification of the chromatographic conditions: UPLC column Hypersil Gold, 100 x $2.1 \mathrm{~mm}, 1.9 \mathrm{~mm}$; gradient $20-30 \% \mathrm{MeOH}$ and $3-8 \% \mathrm{MeCN}(0-8 \mathrm{~min})$, gradient $30-40 \% \mathrm{MeOH}$ and $8-15 \% \mathrm{MeCN}(8$ - $12 \mathrm{~min})$, followed by isocratic $(13-15.5 \mathrm{~min})$ performance in water buffered with $5 \mathrm{mM}$ ammonium formate.

The mass spectrometric data were acquired through the following MRM transitions: JH III $(267 \rightarrow 235)$, JH II $(281 \rightarrow 249)$, JH I $(295 \rightarrow 263), \delta$-tocopherol $(403 \rightarrow 137), \beta-$ and $\gamma$-tocopherols $(417 \rightarrow 151)$, and $\alpha$-tocopherol $(431 \rightarrow 165$, 
see Table 1 below). Specific product ions were monitored, resulting from precursor ions selected according to their characteristic mass-to-charge ratio and retention time.

Table 1. Technical data for identification of separate juvenile hormones and tocopherols.

\begin{tabular}{|l|c|c|c|}
\hline Compound & $\begin{array}{c}\text { Precursor Ion } \\
(\mathrm{m} / \mathrm{z})\end{array}$ & $\begin{array}{c}\text { Product Ion } \\
(\mathrm{m} / \mathrm{z})\end{array}$ & $\begin{array}{c}\text { Retention Time } \\
(\mathrm{min})\end{array}$ \\
\hline JH III & 267 & 235 & 4.4 \\
\hline JH II & 281 & 249 & 4.9 \\
\hline JH I & 295 & 263 & 5.4 \\
\hline$\alpha$-Tocopherol & 403 & 137 & 12.8 \\
\hline $\begin{array}{l}\beta \text { - and } \gamma \text { - } \\
\text { Tocopherol }\end{array}$ & 417 & 151 & 13.6 \\
\hline$\delta$-Tocopherol & 431 & 165 & 14.5 \\
\hline
\end{tabular}

Methods used for chromatography/mass spectrometry analysis of Vitamin E and $\mathrm{JH}-\mathrm{I}$ in a single run were elaborated as part of the postdoctoral dissertation work of J. Paroulek. The values determined for the content of Vitamin E complex and JH-I to JH-III in Figures 1 to 5 were obtained by integration of the chromatographic peaks, using Xcalibur software supplied with the mass spectrometer. The final values were derived from the peaks obtained with the respective external standards. The contents were calculated and expressed per individual organ of the male reproductive system or isolated female abdomen. The amount of tissue in the male reproductive organs was determined by the size of the respective organs. The male fat body, which was contaminated with approximately $25 \%$ of haemolymph, was dissected in the amounts approximately equivalent to one third of the total fat body. The identities of analytes on the chromatograms were confirmed by the respective peaks of the internal standards at the level of 2 ng per sample.

Due to the limited availability of the rare experimental specimens, we present the data in the form of simple average values. The results are based on chromatographic analysis of extracts prepared from reproductive organs dissected from 2 mated and 2 unmated adult males, plus extracts prepared from isolated abdomens of 3 mated and 3 unmated females. Results obtained with two or three equivalent samples of each group were surprisingly similar and, therefore, we give only an average value for each experimental group.

\section{Results}

Development of the standardized chromatographic conditions

Due to its relatively high sensitivity, we used routine analysis of JH-active compounds developed in our laboratory, although it did not allow us reliable separation of $\beta$ - and $\gamma$-tocopherols by chromatography or molecular mass. For 
this reason, we show both these compounds in one chromatographic peak, without being able to specify whether there was only $\beta$ - or $\gamma$-tocopherol or both. Positions of the investigated peaks and their retention times given in Table 1 and Figures 2 and 3, document that the described method can be conveniently used for separation of both JH-related compounds and tocopherols in a single chromatographic run. Figure 2 shows exact positions of the respective chromatographic peaks produced by 2 ng samples of the pure chromatographic standards.

Figure 3 provides a practical example of the chromatographic separation of JH-I, JH-II and JH-III and $\alpha-, \beta$-, $\gamma$ - and $\sigma$-tocopherols found in the extract prepared from abdomens of mated females of $H$. cecropia. The actual concentrations and sizes of the chromatographic peaks can be derived from the respective scales of the separate chromatograms, which were adjusted to fit the size of each peak. For example, the peak of $\alpha$-tocopherol corresponds to a scale of $0-18000$, while the peak of $\sigma$-tocopherol has been displaced on a much smaller scale of 0 to 100 units. Similarly, the peak of JH-I corresponds to a scale of $0-1000$ units, whereas the peak of JH-III is barely visible on the 0 to 150 unit scale. The results in Figure 3 show that the most abundant $\mathrm{JH}$-active compound extracted from the abdomens of the mated females was the epoxyhomofarnesoate ester ( JH-I) and the most abundant derivative of Vitamin $\mathrm{E}$ in the mated females was $\alpha$-tocopherol.

The content of JH-II in the mated females (Figure 3) was profoundly smaller in comparison with the content of JH-I, while JH-III was almost absent or occurred near the lower detection limit (upper left record in Figure 3). Relative abundance of JH-active compounds, calculated from the chromatographic data obtained with the extracts from three abdomens of the mated females, plus data obtained from reproductive organs of four adult males, revealed proportions of JH-I: JH-II: JH-III equivalent to $64000: 100: 20$, respectively. Thus, because the content of JH-active compounds other than JH-I was so small and in the case of JH-III variable, we refrain from presenting special graphs for JH-II and JH-III here.

The relative abundance of individual tocopherol fractions, calculated from the data obtained with pooled extracts of six female abdomens and four male reproductive organs, revealed proportions of $\alpha$-tocopherol, to $\beta+\gamma$-tocopherols, to $\delta$-tocopherol equal to $18400: 550: 100$, respectively. Abdomens of virgin females contained the sesquiterpenoid tocopherols but virtualy no JH-active compounds, suggesting that all JH-I found in the mated females was supplied by ejaculate from the males. 


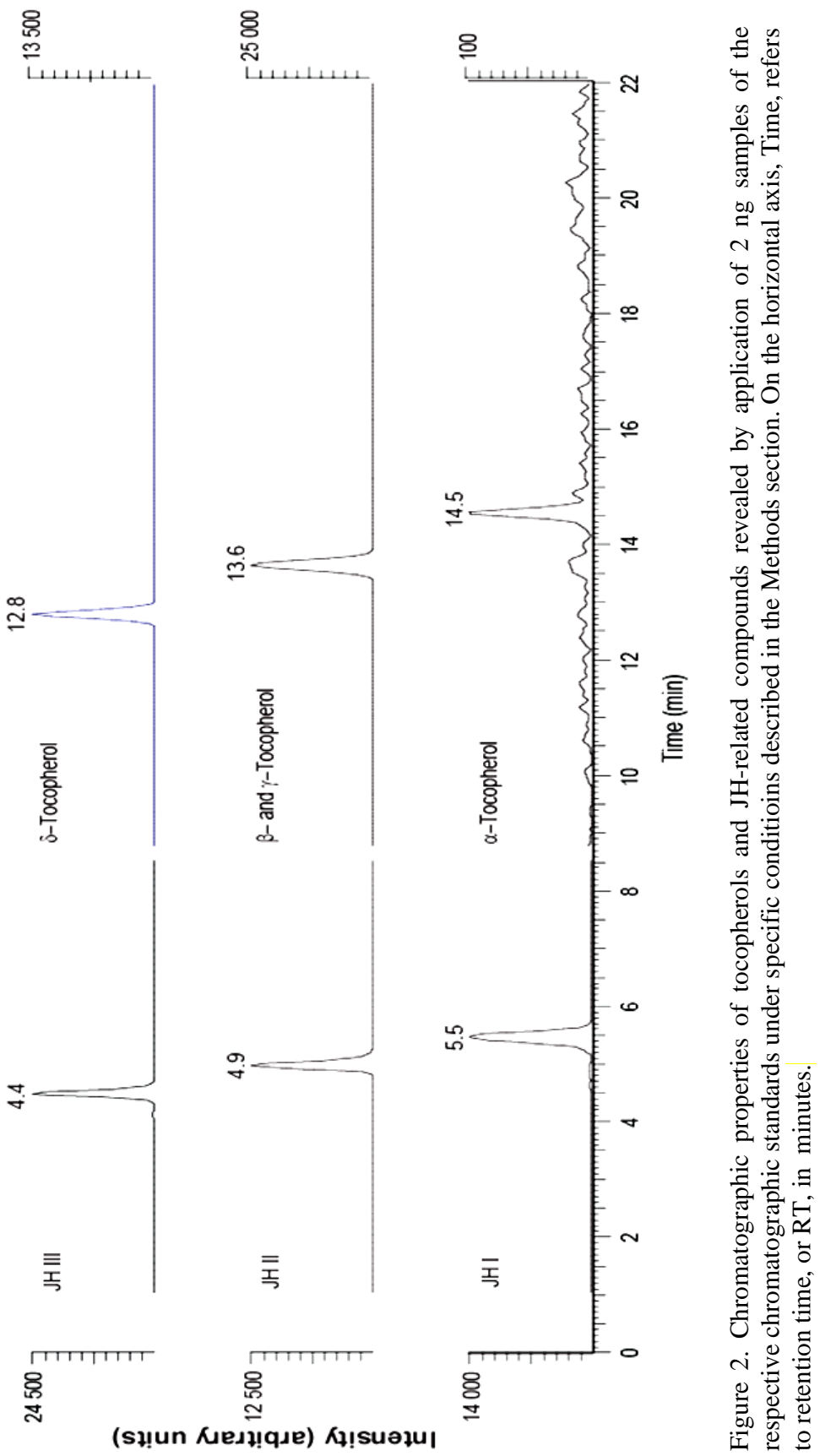




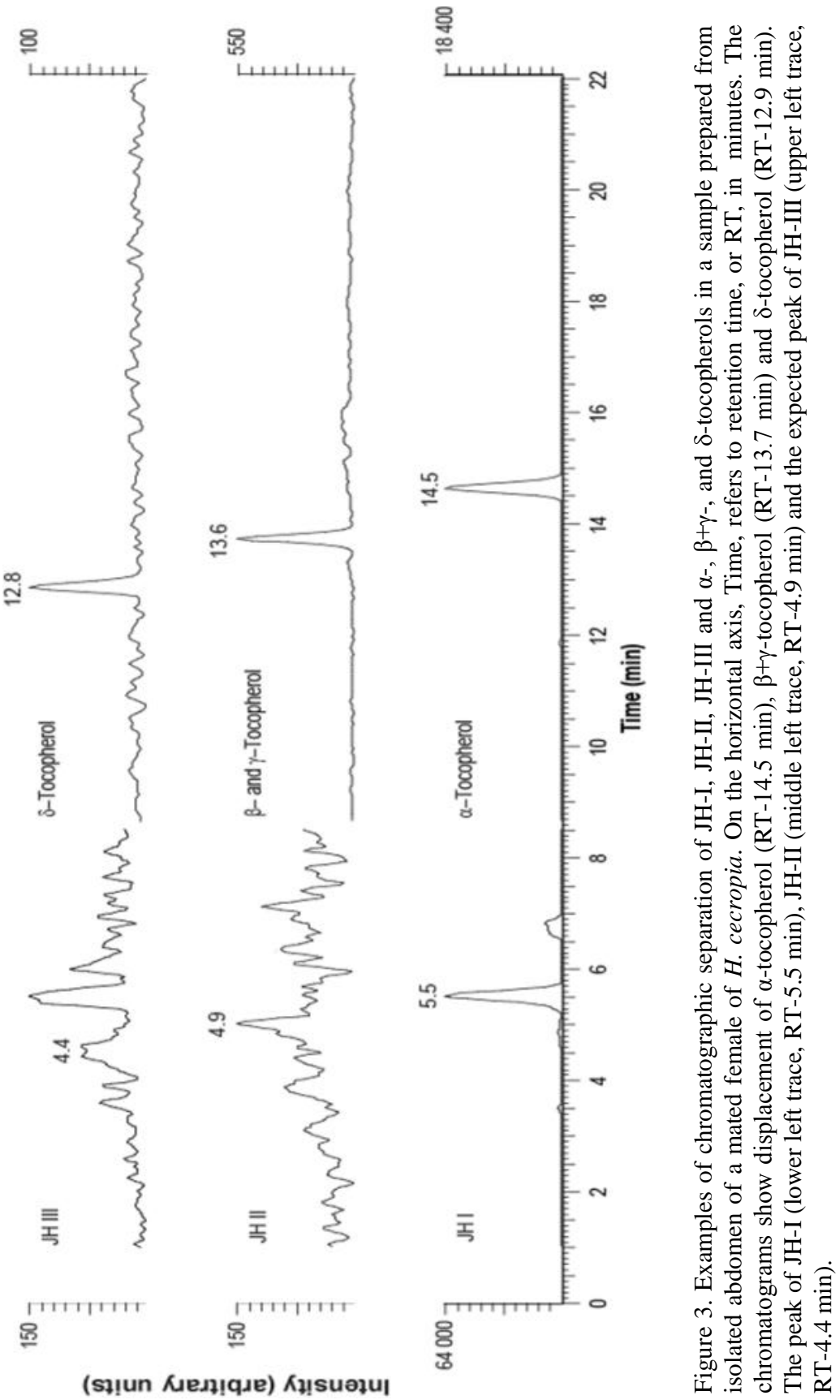


The content of Vitamin $E$ in adult males and females of $H$. cecropia

The results of our chromatographic analysis of $\alpha$-tocopherol, which was the most abundant derivative of Vitamin E, are shown in Figure 4. The presence of $\alpha$-tocopherol and other investigated forms of Vitamin $\mathrm{E}$ (see below) in the abdomens of virgin females suggests two possibilities related to the origin or biosynthesis of Vitamin E in H. cecropia: a) Vitamin E was received earlier with larval food and was stored in the body during the whole period of metamorphosis until its final utilisation in the reproducing adult stage, or; b) Vitamin E could be synthesized de novo from the pool of isoprenoid compounds during later periods of metamorphosis and adulthood. These alternatives may also apply to the content of Vitamin $\mathrm{E}$ in the fat body and reproductive organs of adult males (Figure 4). The greatest amount of $\alpha$-tocopherol was located in the fat body of unmated males (30 ng). We cannot exclude a possibility, however, that $\alpha$-tocopherol was also present in the haemolymph, due to haemolymph contamination of the male fat body. Curiously enough, the content of $\alpha$ tocopherol in the male fat body and haemolymph fraction decreased to almost one third of its original value (10 ng) after the mating (Figure 4). This suggests that the resources of $\alpha$-tocopherol in the fat body or haemolymh could be utilised and transferred into the female body during courtship. This view is supported in Figure 3 by an increased content of $\alpha$-tocopherol in the abdomens of mated females.

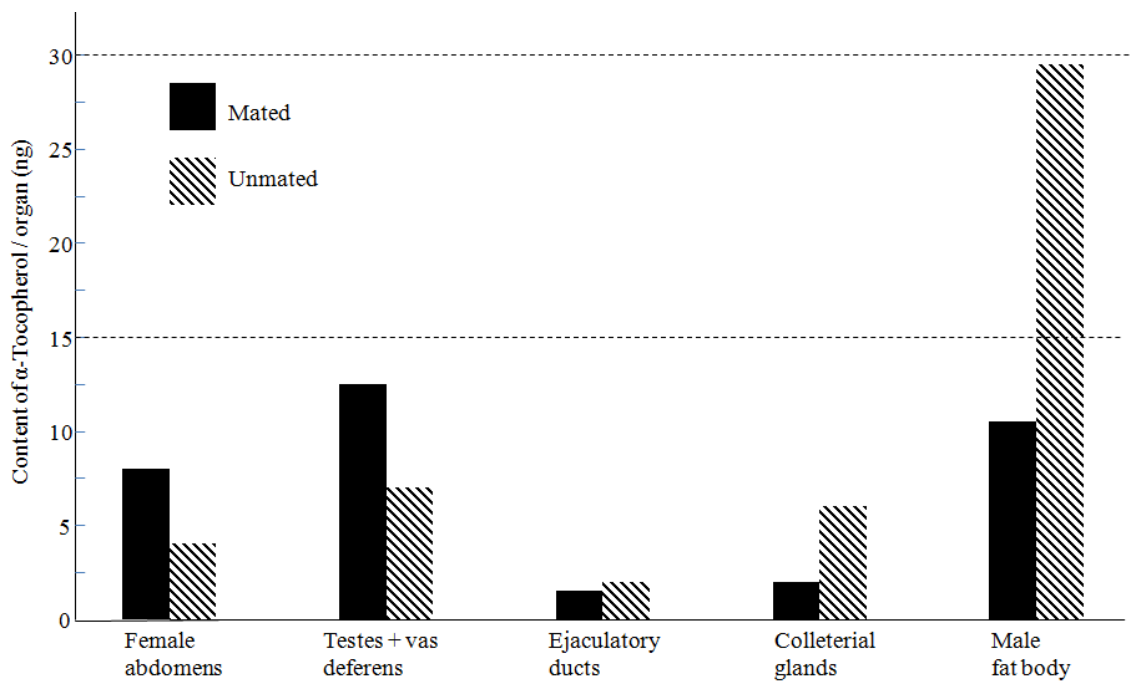

Figure 4. The content of $\alpha$-tocopherol in extracts prepared from isolated female abdomens and reproductive organs of the adult males of $H$. cecropia, sacrificed 3 days after adult emergence, before (in hatched bars) and after mating (in black bars). Mated females were sacrificed before the egg laying. 
The results of our chromatographic analysis in Figure 4 indicate the presence of $\alpha$-tocopherol in all parts of the male reproductive system. The content decreased after mating, except for the relatively high content of $\alpha$ tocopherol in the testes and vas deferens, for which we have no reasonable explanation so far.

We find extensive information about the general importance of Vitamin $\mathrm{E}$ for animal reproduction, which is usually ascribed to its antioxidant properties in the literature. We have already mentioned that various sesquiterpenoid compounds related to $\alpha$-tocopherol can be regularly obtained from plant food (phytyl side chains of chlorophyll). The nonfeeding adult stages of Cecropia moths emerge with fully developed ovaries, testes, ejaculatory ducts containing spermatozoids, and fully formed accessory sexual glands. Therefore, the entire adult reproductive sytem develops long before the adult emergence, during the nonfeeding pupal stage.

The described chromatographic analysis of $\alpha$-tocopherol was accompanied by corresponding analysis of the $\beta$ - and $\gamma$-tocopherols and the less abundant $\delta$ tocopherol. The relative contents of $\beta$ - and $\gamma$-tocopherols were substantially smaller in comparison with the content of $\alpha$-tocopherol shown in Figure 4 . In spite of the quantitative difference, however, Figure 4 shows that the $\beta$ - and $\gamma$ tocopherol fraction revealed a similar distribution in the male and female samples of $H$. cecropia as shown for $\alpha$-tocopherol in Figure 3. In accordance with the data found for $\alpha$-tocopherol, the largest concentrations of $\beta$ - and $\gamma$ tocopherols ( $0.5 \mathrm{ng} /$ organ) were also found in the male fat body (Figure 5$)$. We thus have good reason to assume that physiological processes associated with storage, accumulation, or utilisation of different derivatives of the Vitamin $\mathrm{E}$ complex most likely follow a similar biochemical route.

The above statements related to $\alpha$ - and $\beta+\gamma$-tocopherols may be true for the content and distribution of $\delta$-tocopherol, which was present in relatively small and variable amounts (Figure 3 for comparison). When related to the most abundant $\alpha$-tocopherol, for example, the average contents of $\beta$ - and $\gamma$ tocopherols, calculated the on basis of pooled data obtained from all investigated samples, were $2.96 \%$, while the content of $\delta$-tocopherol in these calculations was less than $0.5 \%$. It has to be added, finally, that the results in Figures 2 to 5 were related only to the tocopherol series of the Vitamin E complex, which is characterised by the saturated sesquiterpenoid side chains. The structurally related series of sesquiterpenoid tocotrienols, containing the unsaturated 2,6,10trienoic sesquiterpenoid side chains (related closely to JH-I) were investigated only qualitatively due to a lack of pure chromatographic standards. 


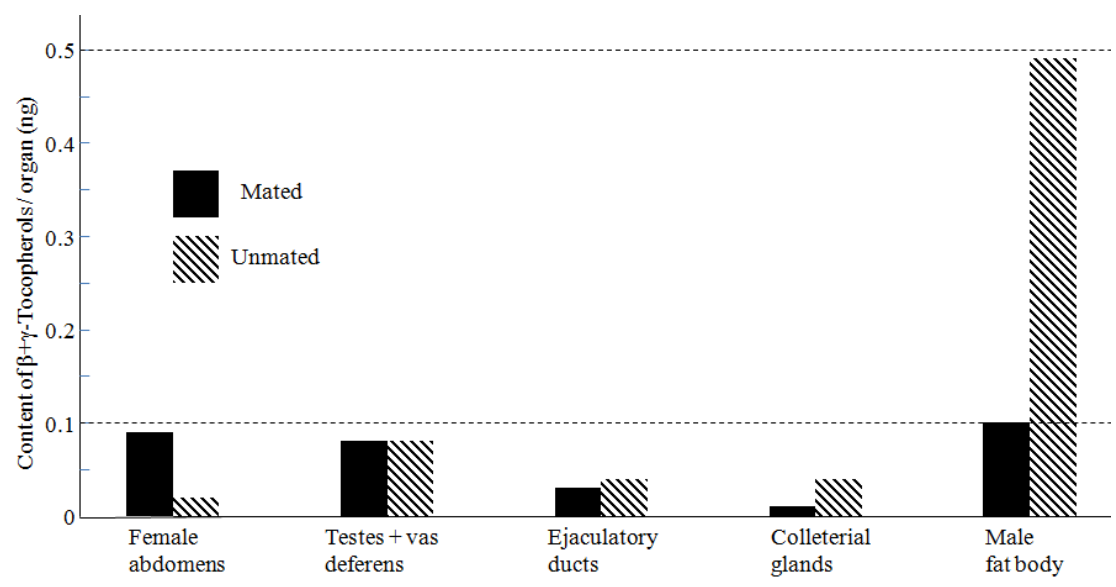

Figure 5. The content of $\beta$ - and $\gamma$-tocopherols in extracts prepared from isolated female abdomens and reproductive organs of the adult males of $H$. cecropia, sacrificed 3 days after adult emergence, before and after mating.

The amounts of JH-related (JH-I, JH-II and JH-III) in adult moths of H. cecropia

The results of our chromatographic analysis of the sesquiteropenoid, JHactive compounds (Figure 6) revealed important physiological features related to the nature of JH-I. The main points can be briefly described as follows: 1 . JH-I, JH-II and JH-III were completely absent or occurred below detectable limits in the bodies (isolated abdomens) of the virgin females; 2 . In contrast to Vitamin E, the JH-active compounds were also completely absent or undetectable in the samples of fat body (containing at least 25\% haemolymph) of the mated or unmated adult males; 3 . The most profound and actually the exclusive source of JH-I was the accessory sexual (colleterial) glands of the unmated males (50 ng/ organ); 4 . The content of JH-I in the colleterial glands substantially diminished (9 $\mathrm{ng} /$ organ) after mating; 5. During successful courtship, substantial amounts of JH-I were translocated from the male colleterial gland into female abdomens, apparently in the form of ejaculate (20 $\mathrm{ng} /$ abdomen); 6. In addition to colleterial glands, other parts of the male reproductive system (testes, vas deferens, ejaculatory ducts) also contained small amounts of JH-I due to their mechanical interconnection with the colleterial glands; 7. JH-I almost completely disappeared from the lumen of vasa deferentia and ejaculatory ducts after mating (Figure 6).

The described results show that the sesquiterpenoid, epoxyhomofarnesoate (JH-I), considered for 50 years to be the true hormone of lepidopteran corpus allatum, may be an excretory product of the exocrine colleterial gland. The related sesquiterpenoids, JH-II and JH-III, were also found in the colleterial glands of male Cecropia silkworms, although they 
occurred in substantially smaller amounts. For example, the colleterial glands of unmated males of Cecropia contained 12-fold less JH-II than JH-I, and the usually most abundant JH-III (methyl 10,11-epoxyfarnesoate), occurred in very small amounts, in most cases under detectable analytical limits.

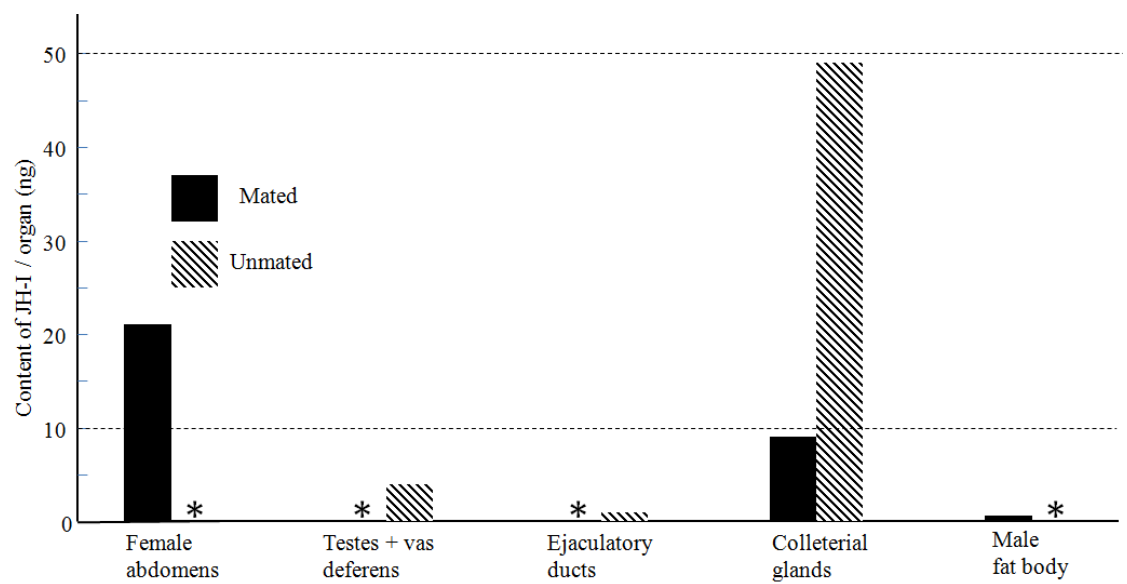

Figure 6. The content of sesquiterpenoid JH-I (methyl 10,11-epoxy, 7-ethyl, 3,11dimethyl, 2,6-tridecadienoate) in extracts prepared from isolated female abdomens and reproductive organs of adult male $H$. cecropia, sacrificed 3 days after adult emergence, before and after mating.

Exact reasons why the male colleterial gland should accumulate isoprenoid excretory products like Vitamin E and produce JH-I, which is absent from fat body and haemolymph, are not known. Dissections (10 years ago) of developing pupae confirmed the previously known literature data that growth and development of the female (ovaries, bursa copulatrix) as well as the male (testes, ejaculatory ducts, colleterial glands) reproductive organs are terminated long before adult emergence, during the earlier period of metamorphosis. This developmental period is characterised in all insect groups by virtual inactivity of the corpora allata and no known action of JH (see discussion). These facts prompted us to conclude, therefore, that the observed exclusive presence of JH-I in the colleterial gland was due to its biosynthesis within the gland, not by its passive transport into the gland from the apparently inactive corpora allata. The statement is in agreement with the virtual absence of JH-I from the male fat body/haemolymph fraction (see Figure 6).

Physiological reasons for the accumulation of sesquiterpenoid Vitamin E and JH-I in the male ejaculate are not completely clear and require further experimental study. The selective advantages of sesquiterpenoids in insect reproduction can be envisaged in antioxidant properties, prolongation of sperm survival, fertilisation, tanning of egg chorion, antimicrobial properties or prolonged survival of the fertilized eggs (see the references below). Our results 
suggest that the correct physiological status of the epoxy-homofarnesoate (JH-I) can be determined as the excretory product of the exocrine colleterial gland of male Cecropia silkworms. The exocrine biological nature of JH-I is fundamentally different from the currently reported endocrine status of the corpus allatum hormone. It is obvious that a correct resolution of endocrine (corpus allatum) or exocrine (colleterial gland) origin of JH-I may be important for future progress in insect endocrinology with \pm 4000 "hormonomimetic" juvenoids (Sláma, 1999, 2013).

\section{Discussion}

The presence of Vitamin E in adult Cecropia silkworms

The biological importance of Vitamin $\mathrm{E}$ is usually ascribed to its role in reproductive functions of humans and other vertebrate animals. The responsible physiological mechanisms are mostly associated with antioxidant properties of Vitamin $\mathrm{E}$ and its protective role over the external or internal cellular membranes (reviewed by Brigelius-Flohé and Traber, 1999; Preedy and Watson, 2007). Recently it has been found, however, that certain derivatives of Vitamin $E$ also play an essential role in regulation of reproductive functions in invertebrates, such as insects (reviewed by Jedlička, 2011). For example, reproducing adult females of Pyrrhocoris apterus developed relatively large accessory sexual glands (ASG) whose secretions were important for determination of properties of the egg shells and influenced survival of the eggs (Jedlička et al., 2009). The ASG of reproducing females grew in size and produced a yellow oily exocrine secretion in response to endogenous hormone secreted by the corpora allata. The secretion contained diacylglycerols of various fatty acids and Vitamin E. Diapausing females with a physiologically inhibited endocrine system or artificially removed sources of JH (allatectomised females), had undeveloped ovaries and small, rudimentary ASG without secretory products. The JH-activated ASG of reproducing females contained predominantly the $\gamma$ - and $\delta$-tocopherol derivatives of the Vitamin E complex. The frequent association of Vitamin $\mathrm{E}$ with reproductive processes in very different groups of animals as are vertebrates and invertebrates, suggests that the assumed antioxidant, antimicrobial or antimutagenic properties of these lipid soluble vitamins with sesquiterpenoid side-chains play an important role in the regulation of reproduction of all animals (Jedlička et al., 2009).

The dependence of accessory sexual glands on $\mathrm{JH}$, as found in the females of Pyrrhocoris (Jedlička et al., 2009), was earlier reported from other insect species (Bodenstein and Shaaya, 1968; Shaaya and Bodenstein, 1969; Williams, 1959, 1963; Sláma et al., 1974; reviewed by Engelman, 1970). In all these instances, however, the effects of $\mathrm{JH}$ were strictly limited to feeding stages. In contrast to this, colleterial glands of Cecropia males grow and develop in a nonfeeding insect stage, where the involvement of endocrine regulations by corpora allata should not be expected (Pflugfelder, 1958; Novák, 1959, 1966, 
1976). Some authors assumed that the colleterial glands of nonfeeding Cecropia silkworms, similarly to the ASG of Pyrrhocoris (Sláma et al., 1974; Jedlička et al., 2009), functioned also under the influence of the hormone from the corpora allata (Meyer et al., 1965), which is apparently in conflict with the fact that the corpus allatum of insects never secretes the hormone in a nonfeeding stage (Bounhiol, 1938; Novák, 1966; Sláma et al., 1974). Accordingly, it was previously found that adult silkworms, Bombyx mori (Linnaeus, 1758), had inactive corpora allata, whose removal had no effect on reproductive physiology of this nonfeeding adult stage (Yamashita et al., 1961; Banno and Akai, 1988). The adult stage of silkworms forms a terminal developmental stage where hormonal regulations by JH are obsolete (reviewed by Hinton, 1951).

When compared with the female ASG of Pyrrhocoris (Jedlička et al., 2009), the male colleterial glands of $H$. cecropia contain a substantially different composition of separate Vitamin E derivatives. Namely, the ASG of Pyrrhocoris contain predominantly $\gamma$ - and $\delta$-tocopherols, while the colleterial glands of Cecropia (Figures 3 and 4) contain mostly $\alpha$-tocopherol. We assume that the difference may be related to a substantially different composition of the food. The caterpillars of $H$. cecropia feed on green leaves of certain fruit trees that are rich in $\alpha$-tocopherol (Munné-Bosch and Alegre, 1990). On the other hand, the predominating content of $\gamma$ - and $\delta$-tocopherols in P. apterus may be due to rather high content of these two forms of Vitamin $\mathrm{E}$ in their linden seed diet (Koštál et al., 2013). This shows that the nature of tocopherol derivatives in different insect species can be influenced by the composition of the plant food they consume.

Jedlička et al. (2009) mention several examples in which $\alpha$-tocopherol can be responsible for increased fecundity of adult moths due to stimulation of ovarian growth. The presence of JH-I and $\alpha$-tocopherol in the ejaculate corresponds well with the frequently reported actions of Vitamin E, i. e. prolongation of sperm survival, stabilization of biomembranes, inhibition of lipid peroxidation, protection of tissues against singlet oxygen and stabilization of ultrastructural formations (Atkinson et al., 2008). These features prevent oxidative damage of sperm stored in the spermatheca and facilitate egg fertilization. In addition to the described role of tocopherols in insect reproduction, Koštál et al. (2013) recently reported on essential functions of these compounds in the membranes of the fat body cells and thoracic muscle cells of $P$. apterus adults during the overwintering period.

A possible de novo biosynthesis of Vitamin $\mathrm{E}$ in insects is still an unresolved problem. It is generally believed that a common source of Vitamin $\mathrm{E}$ in animals is their plant-based diet. In earlier work (Jedlička et al., 2009), we speculated on possible biosynthesis of the tocopherols in the growing and proliferating ASG, together with the biosynthesis of acylglycerols, which do not occur in the haemolymph, fat body and other visceral organs. This situation is somewhat different with respect to colleterial glands of adult Cecropia, which 
can probably make use of the relatively large depot of $\alpha$-tocopherol in male fat body (Figure 4). It is possible that similar biochemical transformations can occur also in females, which contain considerable amounts of $\alpha$-tocopherol before they mate. The present results clearly indicate, however, that the essential sesquiterpenoid compounds (Vitamin E and JH-I) can be substantially augmented after reception of the ejaculate.

\section{The role of sesquiterpenoid JH-I in insect reproduction}

The results of our chromatographic analysis in Figure 6 uncovered a new biological status of the sesquiterpenoid JH-I, namely, that it forms (together with the sesquiterpenoid Vitamin E) essential excretory products of the exocrine accessory sexual (colleterial) glands of the male Cecropia silkmoths. This finding explains the reported absence of JH-activity in lipid extracts made from females, including species in which the lipid extracts of males contained the $\mathrm{JH}$ active materials (Gilbert and Schneiderman, 1958, 1961; Meyer et al., 1965, 1968; Rőller and Dahm, 1970; Rőller et al., 1967; Schneiderman and Gilbert, 1964; Schneiderman et al., 1960; Shirk et al., 1976; Sláma, 1971, 1985; Williams, 1959, 1960, 1963). The reported presence of JH in the males and not in the females represented a real physiological puzzle because in most cases the hormone produced by corpora allata regulated the reproductive processes predominantly in the feeding female stages, and, but rarely in the males (reviews by Pflugfelder, 1958; Novák, 1966, 1976; Engelman, 1970; Sláma et al., 1974).

The accumulation of $\mathrm{JH}$-active materials in the male colleterial glands of Cecropia was previously observed by several authors (Gilbert and Schneiderman, 1961; Meyer et al., 1965, 1968; Shirk et al., 1976; Williams, 1963). All of them deeply adhered to the original explanation of Williams, who proposed that $\mathrm{JH}$-active material was made in the corpora allata and later transported and deposited in certain tissues of the abdomen (Williams, 1963). Serious physiological inconsistencies associated with this concept, namely the questions of why only the male and not the female corpora allata should secrete the $\mathrm{JH}$, or what functions the growth hormone could accomplish in the nonfeeding adult male moth. These and other inconsistencies such as, for example, experimental demonstration of virtual inactivity of the corpora allata in adult silkworms (Banno and Akai, 1988; Yamashita et al., 1961) were neglected by biochemists (Devillers, 2013a, 2013b; Jindra et al., 2013; for review see Sláma, 2013).

Recently, 50 years after elucidation of the JH-I structure (Röller and Bjerke, 1965; Röller et al., 1967, 1969), the belief that JH-I is the true corpus allatum hormone is mainly supported by a brief report of Röller and Dahm (1970) based on in vitro cultivation of corpora allata. The Röller group presented extensive biochemical data suggesting that the corpora allata released into haemolymph the biologically inactive $\mathrm{JH}$ acid, which was then reesterified in the male accessory sexual glands by a SAM dependent methyl transferase into 
biologically active JH-I ester (Peter et al., 1979; Peter et al., 1981; Shirk et al., 1983). These biochemical transformations of JH acid by O-methyltransferase and reciprocal hydrolysis of JH-I ester by esterase enzymes have been frequent subjects of numerous review articles (Tobe and Stay, 1985; Goodman and Granger, 2005; Hui et al., 2013). Unfortunately, insect hormones were treated in the same way as enzymes, without respect to the fact that hormones were still biologically active at concentrations down to $10^{-9} \mathrm{M}$, which is absolutely out of the reach $\left(10^{-6} \mathrm{M}\right)$ of the hydrolytic enzymes (Sláma et al., 1993).

The results of our study cannot directly disprove the tentative possibility that the JH-I would be a true $\mathrm{JH}$ of the corpus allatum. We are convinced, however, that this axiomatic belief is highly improbable. Endocrinological reasons for this statement can be summarised into the following points: 1.Virgin females of Cecropia moths contain corpora allata of similar anatomical structure to those of the males, albeit there is no JH-I in their bodies; 2. JH-I has been encountered only in the male colleterial glands and the associated male reproductive system, not in the fat body and haemolymph; 3. Colleterial glands are exocrine glands producing excretory products into the lumen of the gland; in Cecropia they develop and produce JH-I during metamorphosis, when the corpora allata of all insects are inactive; 4. JH-I could hardly be transferred from corpora allata as $\mathrm{JH}$-acid through haemolymph and transformed into the biologically active hormone in the colleterial glands, which never release their excretory products into the blood; 5. Development of reproductive organs of nonfeeding adults of Cecropia silkworms was terminated long before adult emergence indicating no need for involvement of hormone from the corpora allata; 6. JH-I and Vitamin E become depleted from the lumen of the colleterial glands during courtship, suggesting that JH-I fulfills some important physiological functions in the female reproductive system; 7. Corpora allata never produce $\mathrm{JH}$ in a nonfeeding stage, such as are adult silkworms; 8. Accumulation of JH-I in the lumen of the male colleterial glands is in conflict with animal hormones, which never accumulate in their peripheral targets; 9 . Hormones released from the central neuroendocrine system (neurosecretory cells, corpora cardiaca, corpora allata) have superordinated, epigenetic function over the peripheral genes (reviewed by Sláma, 2013); their functions cannot be disturbed by enzymes (e.g. esterase) or subordinated genes (e.g. Met) of the peripheral target cells. These points reflect the basic problems of $\mathrm{JH}-\mathrm{I}$ identity, which could hardly be altered by increasing the number of investigated specimens.

According to the above described results and discussions, the physiological status of JH-I does not fit the definition of an animal hormone. A hormone is secreted into circulating blood by highly specialized endocrine glands that are used to regulate growth or metabolism in the distant tissue or organs (Hanc, 1959; Gersch, 1964). Our results show that JH-I produced and released into the 
lumen of an exocrine colleterial gland, represents excretory product which is never released into the haemolymph.

Long before isolation of JH-I from lipid extracts and identification of its chemical structure (Rőller and Bjerke, 1965; Rőller et al., 1967), we expressed serious endocrinological arguments against the corpus allatum nature of the Cecropia extracts. The doubts were later increased by the discovery of false juvenile, or JH-mimetic effects of the lipid extracts (Sláma, 1961, 1962) from microorganisms, plants, various vertebrate organs and even from ordinary cream (reviews by Schneiderman and Gilbert, 1964; Sláma et al., 1 974). During the rush for JH analogues (1970 to 1990; reviews by Sláma, 1985, 1999; Sláma et al., 1974), the arguments against the belief that JH-I was the true corpus allatum hormone were further increased by recognition that certain man-made peptidic analogues of JH were more than million-fold more active than JH-I, which was notoriously believed to be the true JH (Sláma, 1999; Sláma et al., 1974).

The most recent reviews on insect JH still propagate the original, 50-yearold concepts in statements like: "The juvenile hormones of insects are sesquiterpenoid molecules secreted by the corpora allata " (Devillers, 2013b), as well as by similar statements that can be found in a number of textbooks and review articles (Nijhout, 1884, Klowden, 2007; Gilbert, 2009; Riddiford, 1996, 2008, 2012; Jindra et al., 2013; Devillers, 2013a,b). Existence of more than 4000 sesquiterpenoid and other JH mimics (Sláma, 1999) and those described here, exocrine, not endocrine, nature of JH-I, point out that the old dogmatic views on the nature and mode of action of insect corpus allatum hormone need to be carefully reinvestigated (Sláma, 2013).

\section{Acknowledgment}

We are indebted to Dr. P. Jedlička for technical help and preparation and to several anonymous reviewers for their constructive recommendations.

\section{Literature Cited}

Atkinson, J., R. F. Epand, R. M. Epand. 2008. Tocopherols and tocotrienols in membranes: a critical view. Free Radical Biology and Medicine 44:739-764. http://dx.doi.org/10.1016/j.freeradbiomed.2007.11.010

Banno, Y. and H. Akai. 1988. Ultrastructural changes of corpus allatum in the imaginal stages in the silkworm, Bombyx mori. Journal of Sericultural Science of Japan 57:431-437.

Bodenstein, D. and E. Shaaya. 1968. The function of the accessory sex glands in Periplaneta americana (L.). A quantitative bioassay for juvenile hormone. Proceedings of National Academy of Sciences of the United States of America 59: 1279-1290. http://dx.doi.org/10.1073/pnas.59.4.1223

Bounhiol, J. J. 1938. Recherches expérimentales sur le déterminisme de la métamorphose chez les Lépidoptères. Bulletin Biologique France Belgique 24:1-199.

Brigelius-Flohé, R. and M. G. Traber. 1999. Vitamin E: function and metabolism. The FASEB Journal (Federation of American Societies for Experimental Biology) 13:1145-1155.

De Loof, A., B. Boerjan, U. R. Ernst, and L. Schoofs. 2013. The mode of action of juvenile hormone and ecdysone: Towards an epi-endocrinological paradigm? General and Comparative Endocrinology 188:35-45. http://dx.doi.org/10.1016/j.ygcen.2013.02.004

De Loof, A., W. De Haes, T. Janssen, and L. Schoofs. 2014. The essence of insect metamorphosis and aging: Electrical rewiring of cells driven by the principles of juvenile hormone-dependent 
Ca2+-homeostasis. General and Comparatiave Endocrinology 199: 70-85. http://dx.doi.org/10.1016/j.ygcen.2014.01.009

Devillers, J. 2013a. Juvenile hormones and juvenoids. Modeling biologial effects and environmental fate. CRC Press, Taylor and Francis Group. Boca Raton, Florida, USA. 387pp.

Devillers, J. 2013b. Juvenile hormones and juvenoids: A historical survey. pp. 1-14. In, J. Devillers (Editor). Juvenile hormones and juvenoids. Modeling biologial effects and environmental fate. CRC Press, Taylor and Francis Group. Boca Raton, Florida, USA. 387pp.

Engelman, F. 1970. The Physiology of Insect Reproduction. Pergamon Press, Inc. Oxford, England, UK. 307 pp.

Gersch, M. 1964. Vergleichende Endocrinologie der wirbellosen Tiere. Academische Verlagsgesellschaft Geest and Portig. K.-G. Leipzig, German Democratic Republic (East Germany). 535 pp.

Gilbert, L. I. 2009. Insect Development:Morphogenesis, Molting and Metamorphosis. Academic Press, Elsevier BV. London, England, UK. 739 pp.

Gilbert, L. I. and H. A. Schneiderman. 1958. The occurence of substances with juvenile hormoneactivity in adrenal cortex of vertebrates. Science (Washington, District of Columbia, USA) 128:844. http://dx.doi.org/10.1016/0016-6480(61)90008-9

Gilbert L. I. and H. A. Schneiderman. 1961. The content of juvenile hormone and lipid in Lepidoptera. Comparataive Endocrinology 1:453-472. http://dx.doi.org/10.1016/0016$\underline{6480(61) 90008-9}$

Goodman, W. and N. Granger. 2005. The juvenile hormones. pp. 319-408. In, Gilbert, L.I., K. Iatrou, and S. S. Gill (Editors). Comprehensive Molecular Insect Science. Elsevier Pergamon. Oxford, England, UK. Volume 3. 842 pp. http://dx.doi.org/10.1016/B0-44-451924-6/00039-9

Hanc, O. 1959. Hormone. VEB Gustav Fischer Verlag. Jena, German Democratic Republic (East Germany). 633pp.

Hinton, H. E. 1951. The stucture and function of the endocrine glands of the Lepidoptera. Proceedings of South London Entomological and Natural History Society 1951:124-160.

Hui, J. H. L. W. G. Bendena, and S. S. Tobe. 2013. Future perspectives for research on the biosynthesis of juvenile hormones and related sesquiterpenoids in arthropod endocrinology and ecotoxicology. pp. 15-30. In, J. Devillers (Editor). Juvenile hormones and juvenoids. Modeling biologial effects and environmental fate. CRC Press, Taylor and Francis Group. Boca Raton, Florida, USA. 387 pp.

Jedlička, P. 2011. Present state of knowledge about research on vitamin E in insects (review). In, Lindberg A. E. (Editor). Vitamin E: Nutrition, Side Effects and Supplements. Nova Science Publishers. Hauppauge, New York, USA. pp. 303-308.

Jedlička, P., J. Cvačka and K. Sláma, 2009. Juvenile hormone-stimulated synthesis of acyl-glycerols and Vitamin E in female accessory sexual glands of the fire bug, Pyrrhocoris apterus L. Archives of Insect Biochemistry and Physiology 72: 48-59. http://dx.doi.org/10.1002/arch.20322

Jindra M., S. R. Palli, and L. M. Riddiford. 2013. The juvenile hormone signaling pathway in insect development. Annual Reviews of Entomology 58: 181-204. http://dx.doi.org/10.1146/annurevento-120811-153700

Karlson, P. anf P. Schmialek, 1959. Nachweis der Excretion von Juvenilhormon. Zeitschrift für Naturforschung 14:832.

Klowden, J. 2007. Physiological Systems in Insects. Second Edition. Elsevier. Amsterdam, The Netherlands. 688 pp.

Koštál, V., T. Urban, L. Rimnáčová, P. Berková, and P. Šimek. 2013. Seasonal changes in minor membrane phospholipid classes, sterols and tocopherols in overwintering insect, Pyrrhocoris $\begin{array}{llll}\text { apterus. } & \text { Journal } & \text { Insect }\end{array}$ http://dx.doi.org/10.1016/j.jinsphys.2013.06.008

Meyer, A. S., H. A. Schneiderman, and L. I. Gilbert. 1965. A highly purified preparation of juvenile hormone from the silk moth Hyalophora cecropia L. Nature (London, England, UK) 206:272275. http://dx.doi.org/10.1038/206272a0 
Meyer, A.S., H. A. Schneiderman, E. Hanzmann and J. H. Ko. 1968. The two juvenile hormones from the Cecropia silk moth. Proceedings of National Academy of Sciences of the United States of America 60: 853-860. http://dx.doi.org/10.1073/pnas.60.3.853

Munné-Bosh, S. and L. Alegre, 2002. The function of tocopherols and tocotrienols in plants. Critical Reviews of Plant Science, 21: 31-57. http://dx.doi.org/10.1080/0735-260291044179

Nation, J. L. 2002. Insect Physiology and Biochemistry. CRC Press. Boca Raton, Florida, USA. 485 pp.

Nijhout, H. F. 1994. Insect Hormones. Princeton University Press. Princeton, New Jersey, USA. 267 pp.

Novák, V. J. A. 1959. Insektenhormone (Second Edition). Nakladatelstvi ČSAV. Prague, Czechoslovakia. 283 pp.

Novák, V. J. A. 1966. Insect Hormones. Methuen. London, England, UK. 478 pp.

Novák, V. J. A. 1967. The juvenile hormone and the problem of animal morphogenesis. pp. 119132. In, Insects and Physiology. essays presented to Sir Vincent Wigglesworth, CBE, MD, FRS on his retirement from the Quick Chair of Biology and Directorship of the Unit of Insect Physiology in the University of Cambridge. J. W. Beament, J. E. Treherne, and V. B. Wigglesworth (Editors). Oliver \& Boyd. Edinburgh, Scottland, UK. 378 pp.

Novák, V. J. A. 1976. Insect Hormones (Second English Edition). Chapman and Hall. London, England, UK. 600pp.

Novák, V. J. A. and E. Červenková, 1959. The function of corpus allatum in the last larval instar of metabolic insects. Acta Symposii de Evolutione Insectorum (Prague, Czechoslovakia) Volume 1:152-156.

Pflugfelder, O. 1958. Entwicklungsphysiologie der Insekten. Leipzig, German Democratic Republic (East Germany). 490pp.

Peter, M. G., S. Gunawan, and H. Emmerich, 1979. Preparation of optically pure juvenile hormone I labelled in the ester methyl group with tritium at very high specific activity. Experientia 35:1141-1142. http://dx.doi.org/10.1007/BF01963247

Peter, M. G., P. D. Shirk, K. H. Dahm and H. Röller. 1981. On the specificity of Juvenile Hormone Biosynthesis in the Male Cecropia Moth. Zetschrift für Naturforschung, 36c:579-585.

Preedy, V. R. and R. R. Watson. 2007. The Encyclopedia of Vitamin E. CAB International Publishing, Cromwell Press. Townbridge, England, UK. 962 pp.

Riddiford, L. M. 1996. Juvenile hormone: the status of its "status quo" action. Archives of Insect Biochemistry and Physiology 32: 271-286. http://dx.doi.org/10.1002/(SICI)15206327(1996)32:3/4<271::AID-ARCH2>3.0.CO;2-W

Riddiford, L. M. 2008. Juvenile hormone action. A 2007 perspective. Journal of Insect Physiology 59:895-901. http://dx.doi.org/10.1016/j.jinsphys.2008.01.014

Riddiford, L. M. 2012. How does juvenile hormone control metamorphosis and reproduction? General and Comparative Endocrinology 179: 477-484. http://dx.doi.org/10.1016/j.ygcen.2012.06.001

Röller, H. and J. S. Bjerke. 1965. Purification and isolation of juvenile hormone and its action in lepidopteran larvae. Life Sciences 4:1617-1624. http://dx.doi.org/10.1016/0024-3205(65)90141$\underline{4}$

Röller, H. and K. H. Dahm. 1970. The identity of juvenile hormone produced by corpora allata in vitro. Naturwissenschaften 57: 454-455. http://dx.doi.org/10.1007/BF00607739

Röller, H., K. H. Dahm, C. C. Sweeley, and B. M. Trost. 1967. Die Struktur des Juvenilhormons. Angewandte Chemie 79:190-191. http://dx.doi.org/10.1002/ange.19670790411

Röller, H., J. S. Bjerke, L. M. Holthaus, D. W.Norgard and W. H. McShan,1969. Isolation and biological properties of the juvenile hormone. Journal of Insect Physiology 15:379-389. http://dx.doi.org/10.1016/0022-1910(69)90285-6

Schmialek, P. 1961. Die Identifizierung zweier in Tenebriokot und in Hefe vorkommender Substanzen mit Juvenilhormonwirkung. Zeitschrifft für Naturforschung 16b:461-464.

Schmialek, P. 1963. Über die Bildung von Juvenilhormonen in Wildseidenspinnern. Zeitschrifft für Naturforschung 18b:462-465. 
Schneiderman, H. A. and L. I. Gilbert, 1958. Substances with juvenile hormone activity in Crustacea and other invertebrates. Biological Bulletin 115:530-535. http://dx.doi.org/10.2307/1539115

Schneiderman, H. A. and L. I. Gilbert. 1964. Control of growth and development in insects. Science (Washington, District of Columbia, USA) 143:325-333. http://dx.doi.org/10.1126/science.143.3604.325

Schneiderman, H. A., L. I. Gilbert and M. J. Weinstein, 1960. Juvenile hormone activity in microorganisms and plants. Nature (London, England, UK) 188: 1041-1042. http://dx.doi.org/10.1038/1881041a0

Shaaya, E. and D. Bodenstein. 1969. The function of the accessory sex glands in Periplaneta americana (L.) II. The role of the juvenile hormone in the synthesis of protein and protocatechuic acid glucoside. Journal of Experimental Zoology 170: 281-292. http://dx.doi.org/10.1002/jez.1401700305

Shirk, P. D., K. H. Dahm, and H. Röller. 1976. The accessory sex glands as the repository for juvenile hormones in male cecropia moths. Zeitschrift für Naturforschung 31c:199-200.

Shirk, P. D., G. Bhaskaran and H. Roller, 1983. Developmental physiology of corpora allata and accessory sex glands in the Cecropia Silkmoth. Journal of Experimental Zoology 227: 69-79. http://dx.doi.org/10.1002/jez.1402270111

Sláma, K. 1961. Pseudo-juvenilizing effect in insects. Acta societatis entomologicae Čechosloveniae $58: 117-120$.

Sláma, K. 1962. The juvenile hormone-like effect of fatty acids, fatty alcohols and some other compounds in insect metamorphosis. Acta societatis entomologicae Čechosloveniae 59: 323340.

Sláma, K. 1971. Insect juvenile hormone analogues. Annual Reviews of Biochemistry 40:1079-1102. http://dx.doi.org/10.1146/annurev.bi.40.070171.005243

Sláma, K. 1983. Illusive functions of the prothoracic gland in Galleria. Acta entomologica bohemoslovaca 80:160-176.

Sláma, K. 1985. Pharmacology of insect juvenile hormones. pp. 357-394. In, Gilbert, L.I. and B. A. Kerkutt (Editors). Comprehensive Insect Physiology, Biochemistry and Pharmacology. Pergamon Press. Oxford, England, UK. Volume 11: 849 pp.

Sláma, K. 1999. The history and current status of juvenoids. pp. 9-25. In, W. M. Robinson, F. Rettich and G.W. Rambo (Editors). Proceedings of 3rd International Conference on Urban Pests. Hronov, Czech Republic. 678 pp.

Sláma, K. 2013. Insect hormones: more than 50-years after discovery of insect juvenile hormone analogues (JHA, juvenoids). Terrestrial Arthropod Reviews 6:1-77. http://dx.doi.org/10.1163/18749836-06041073

Sláma, K. and J. Lukáš. 2013. Role of juvenile hormone in the hypermetabolic production of water revealed by the $\mathrm{O}_{2}$ consumption and thermovision images of larvae of insects fed a diet of dry food. European Journal of Entomology 110:221-230. http://dx.doi.org/10.14411/eje.2013.032

Sláma, K., M. Romaňuk, and F. Šorm. 1974. Insect Hormones and Bioanalogues. Springer. Wien, Austria. 477pp. http://dx.doi.org/10.1007/978-3-7091-8331-1

Sláma, K., D. Konopinska, and W. Sobotka. 1993. Effect of proctolin on outonomic physiological functionsin insects. European Journal of Entomology 90:23-35.

Tobe, S. S. and B. Stay, 1985. Structure and regulation of the corpus allatum. Advances of Insect Physiology 18:305-432. http://dx.doi.org/10.1016/S0065-2806(08)60043-0

Wigglesworth, V. B. 1957. The action of growth hormones in insects. Symposia of Society for experimental Biology 11:204-227.

Williams, C. M. 1952. Physiology of insect diapause. -IV. The brain and prothoracic glands as an endocrine system in the cecropia silkworm. Biological Bulletin of Woods Hole 103:120-138. http://dx.doi.org/10.2307/1538411

Williams, C. M. 1956. The juvenile hormone of insects. Nature (London, England, UK) 178:212213. http://dx.doi.org/10.1038/178212b0

Williams, C. M. 1959. The juvenile hormone -I. Endocrine activity of the corpora allata of the adult cecropia silkworm. Biological Bulleting of Woods Hole 116:323-338. http://dx.doi.org/10.2307/1539218 
Williams, C. M. 1960. The juvenile hormone. Acta Endocrinologica (Copenhagen, Denmark) 34(Supplementum 50):189-191.

Williams, C. M. 1963. The juvenile hormone -III. Its accumulation and storage in the abdomens of certain male moths. Biological Bulletin Woods Hole 124:355 - 367. http://dx.doi.org/10.2307/1539485

Williams, C. M. 1987. Midgut of lepidopteran pupae is a major depot of sequestered, mobilizable ecdysteroids. Memoirs Institute Oswaldo Cruz 82:47-49. http://dx.doi.org/10.1590/S0074$\underline{02761987000700010}$

Yamashita, Y., K. Tani, and M. Kobayashi. 1961. The effects of allatectomy on the number of eggs in the silkworm, Bombyx mori. Acta Serologica 39:12-15. 